\title{
THREE THEOREMS ON COSYMPLECTIC HYPERSURFACES OF SIX-DIMENSIONAL HERMITIAN SUBMANIFOLDS OF THE CAYLEY ALGEBRA
}

\author{
AHMAD AL-OTHMAN and M. BANARU
}

Received 1 June 2002

\begin{abstract}
It is proved that cosymplectic hypersurfaces of six-dimensional Hermitian submanifolds of the octave algebra are ruled manifolds. A necessary and sufficient condition for a cosymplectic hypersurface of a Hermitian submanifold $M^{6} \subset O$ to be a minimal submanifold of $M^{6}$ is established. It is also proved that a sixdimensional Hermitian submanifold $M^{6} \subset O$ satisfying the $g$-cosymplectic hypersurfaces axiom is a Kählerian manifold.
\end{abstract}

2000 Mathematics Subject Classification: 53C10, 58C05.

1. Introduction. One of the most important properties of a hypersurface of an almost Hermitian manifold is the existence of such a hypersurface of an almost contact metric structure determined in a natural way. This structure has been studied mainly in the case of Kählerian [8, 17] and quasi-Kählerian $[13,15]$ manifolds. In the case the ambient manifold is Hermitian, however, comparatively little is known about the geometry of its hypersurfaces. In the present paper, certain results obtained in this direction by using the Cartan structural equations of such hypersurfaces are given.

2. Preliminaries. We consider an almost Hermitian manifold, that is, a $2 n$ dimensional manifold $M^{2 n}$ with Riemannian metric $g=\langle\cdot, \hbar \hbar\rangle$ and an almost complex structure $J$. Moreover, the following condition must hold:

$$
\langle J X, J Y\rangle=\langle X, Y\rangle, \quad \forall X, Y \in \kappa\left(M^{2 n}\right),
$$

where $\mathrm{N}\left(M^{2 n}\right)$ is the module of smooth vector fields on $M^{2 n}$. All the considered manifolds, tensor fields, and similar objects are assumed to be the class $C^{\infty}$.

The specification of an almost Hermitian structure on a manifold is equivalent to the setting of a $G$-structure, where $G$ is the unitary group $U(n)$ [1]. Its elements are the frames adapted to the structure ( $A$-frames). They look as follows:

$$
\left(p, \varepsilon_{1}, \ldots, \varepsilon_{n}, \varepsilon_{\hat{1}}, \ldots, \varepsilon_{\hat{n}}\right)
$$


where $p \in M^{2 n}, \varepsilon_{a}$ are the eigenvectors corresponding to the eigenvalue $i=$ $\sqrt{-1}$, and $\varepsilon_{\hat{a}}$ are the eigenvectors corresponding to the eigenvalue $-i$. Here the index $a$ ranges from 1 to $n$, and $\hat{a}=a+n$.

Therefore, the matrix of the operator of the almost complex structure written in an $A$-frame looks as follows:

$$
\left(J_{j}^{k}\right)=\left(\frac{i I_{n}}{0} \| \frac{0}{-i I_{n}}\right),
$$

where $I_{n}$ is the identity matrix and $k, j=1, \ldots, n$.

We recall that the fundamental (or Kählerian [2]) form of an almost Hermitian manifold is determined by

$$
F\langle X, Y\rangle=\langle X, J Y\rangle, \quad X, Y \in \aleph\left(M^{2 n}\right) .
$$

By direct computation, it is easy to obtain that in an $A$-frame the fundamental form matrix looks as follows:

$$
\left(F_{k j}\right)=\left(\frac{0}{-i I_{n}} \| \frac{i I_{n}}{0}\right) .
$$

Let $O \equiv R^{8}$ be the Cayley algebra. It is well known that two nonisomorphic three-fold vector cross products are defined on it as (see [10])

$$
\begin{aligned}
& P_{1}(X, Y, Z)=-X(\bar{Y} Z)+\langle X, Y\rangle Z+\langle Y, Z\rangle X-\langle Z, X\rangle Y, \\
& P_{2}(X, Y, Z)=-(X \bar{Y}) Z+\langle X, Y\rangle Z+\langle Y, Z\rangle X-\langle Z, X\rangle Y,
\end{aligned}
$$

where $X, Y, Z \in O,\langle\cdot, \cdot\rangle$ is the scalar product in $O$, and $X \rightarrow \bar{X}$ is the conjugation operator. Moreover, any other threefold vector cross product in the octave algebra is isomorphic to one of the above-mentioned cross products.

If $M^{6} \subset O$ is a six-dimensional oriented submanifold, then the induced almost Hermitian structure $\left\{J_{\theta}, g=\langle\cdot, \cdot\rangle\right\}$ is determined by the relation

$$
J_{\theta}(X)=P_{\theta}\left(X, e_{1}, e_{2}\right), \quad \theta=1,2,
$$

where $\left\{e_{1}, e_{2}\right\}$ is an arbitrary orthonormal basis of the normal space of $M^{6}$ at the point $p, X \in T_{p}\left(M^{6}\right)$ [10]. The submanifold $M^{6} \subset O$ is called Hermitian if the almost Hermitian structure induced on it is integrable. The point $p \in M^{6}$ is called general [11] if

$$
e_{0} \notin T_{p}\left(M^{6}\right), \quad T_{p}\left(M^{6}\right) \subseteq L\left(e_{0}\right)^{\perp},
$$

where $e_{0}$ is the unit of Cayley algebra and $L\left(e_{0}\right)^{\perp}$ is its orthogonal supplement. A submanifold $M^{6} \subset O$ consisting only of general points is called a generaltype submanifold [11]. In what follows, all submanifolds $M^{6}$ to be considered are assumed to be of general type. 
3. Cosymplectic hypersurfaces of Hermitian $M^{6} \subset O$. Let $N$ be an oriented hypersurface of a Hermitian submanifold $M^{6} \subset O$ and let $\sigma$ be the second fundamental of the immersion of $N$ into $M^{6}$. As it is well known [15, 17], the almost Hermitian structure on $M^{6}$ induces an almost contact metric structure on $N$. We recall $[13,15]$ that an almost contact metric structure on the manifold $N$ is defined by the system $\{\Phi, \xi, \eta, g\}$ of tensor fields on this manifold, where $\xi$ is a vector, $\eta$ is a covector, $\Phi$ is a tensor of a type $(1,1)$, and $g$ is a Riemannian metric on $N$ such that

$$
\begin{gathered}
\eta(\xi)=1, \quad \Phi(\xi)=0, \quad \eta \hbar \Phi=0, \quad \Phi^{2}=\mathrm{id}+\xi \otimes \eta, \\
\langle\Phi X, \Phi Y\rangle=\langle X, Y\rangle-\eta(X) \eta(Y), \quad X, Y \in \mathrm{N}(N) .
\end{gathered}
$$

The almost contact metric structure is called cosymplectic [15] if

$$
\nabla \eta=\nabla \Phi=0
$$

(Here $\nabla$ is the Levi-Civita connection of the metric $g$ ). The first group of the Cartan structural equations written in an $A$-frame of a hypersurface of a Hermitian manifold looks as follows [16]:

$$
\begin{aligned}
d \omega^{a}= & \omega_{b}^{a} \Lambda \omega^{b}+B_{c}^{a b} \omega^{c} \Lambda \omega_{b}+\left(\sqrt{2} B_{b}^{a 3}+i \sigma_{b}^{a}\right) \omega^{b} \Lambda \omega \\
& +\left(-\frac{1}{\sqrt{2}} B_{3}^{a b}+i \sigma^{a b}\right) \omega_{b} \Lambda \omega ; \\
d \omega_{a}= & -\omega_{a}^{b} \Lambda \omega_{b}+B_{a b}^{c} \omega_{c} \Lambda \omega^{b}+\left(\sqrt{2} B_{a 3}^{b}-i \sigma_{a}^{b}\right) \omega_{b} \Lambda \omega \\
& +\left(-\frac{1}{\sqrt{2}} B_{a b}^{3}+i \sigma_{a b}\right) \omega^{b} \Lambda \omega ; \\
d \omega= & \left(\sqrt{2} B_{b}^{3 a}-\sqrt{2} B_{3 b}^{a}-2 i \sigma_{b}^{a}\right) \omega^{b} \Lambda \omega_{a}+\left(B_{3 b}^{3}+i \sigma_{3 b}\right) \omega \Lambda \omega^{b} \\
& +\left(B_{3}^{3 b}-i \sigma_{3}^{b}\right) \omega \Lambda \omega_{b} .
\end{aligned}
$$

Here and in what follows, $a, b, c=1,2 ; \hat{a}=a+3 ; \alpha, \beta, \gamma, \mu=1,2,3 ; \varphi=7,8$; $k, j=1,2,3,4,5,6$. Note that $\left\{B_{c}^{a b}\right\}$ and $\left\{B_{a b}^{c}\right\}$ are the components of the Kirichenko structural tensor of the Hermitian manifold. These complex tensors form a complete system of first-order differential-geometrical invariants of an arbitrary almost Hermitian structure [2, 6]. According to [6, definition (1)], the components of the Kirichenko tensors are connected to the components of $\nabla J$ :

$$
B_{c}^{a b}=-\frac{i}{2} J_{\hat{b}, c}^{a}, \quad B_{a b}^{c}=\frac{i}{2} J_{b, \hat{c}}^{\hat{a}}
$$

where these tensors satisfy the equations

$$
\begin{aligned}
& d B_{c}^{a b}+B_{d}^{a b} \omega_{c}^{d}-B_{c}^{d b} \omega_{d}^{a}-B_{c}^{a d} \omega_{d}^{b}=B_{c, k}^{a d} \omega^{k}, \\
& d B_{a b}^{c}-B_{a b}^{d} \omega_{d}^{c}+B_{d b}^{c} \omega_{d}^{a}+B_{a d}^{c} \omega_{b}^{d}=B_{a b, k}^{c} \omega^{k} .
\end{aligned}
$$


with the properties

$$
B_{c}^{a b}+B_{c}^{b a}=0, \quad B_{a b}^{c}+B_{b a}^{c}=0, \quad \bar{B}_{c}^{a b}=B_{a b}^{c}
$$

and they are skew-symmetric on the first pair indices.

Taking into account that the first group of the Cartan structural equations of the cosymplectic structure must look as follows [9]:

$$
\begin{aligned}
d \omega^{a} & =\omega_{b}^{a} \Lambda \omega^{b}, \\
d \omega_{a} & =-\omega_{a}^{b} \Lambda \omega_{b}, \\
d \omega & =0,
\end{aligned}
$$

we get the conditions whose simultaneous fulfillment is a criterion for the hypersurface $N$ to be cosymplectic:

$$
\begin{gathered}
B_{c}^{a b}=0, \\
\sqrt{2} B_{b}^{a 3}+i \sigma_{b}^{a}=0, \\
-\frac{1}{\sqrt{2}} B_{3}^{a b}+i \sigma^{a b}=0, \\
\sqrt{2} B_{b}^{3 a}-\sqrt{2} B_{3 b}^{a}-2 i \sigma_{b}^{a}=0, \\
B_{3}^{3 b}-i \sigma_{3}^{b}=0,
\end{gathered}
$$

and the formulas are obtained by complex conjugation (so there is no need to write them down explicitly).

Now, we analyze the obtained conditions. From (3.8c), it follows that

$$
\sigma^{a b}=-\frac{i}{\sqrt{2}} B_{3}^{a b}
$$

By alternating this relation, we get

$$
0=\sigma^{[a b]}=-\frac{i}{\sqrt{2}} B_{3}^{[a b]}=-\frac{i}{\sqrt{2}}\left(B_{3}^{a b}-B_{3}^{b a}\right)=-\frac{i}{\sqrt{2}} B_{3}^{a b} .
$$

Therefore, $B_{3}^{a b}=0$ and consequently $\sigma^{a b}=0$. From (3.8b), we get that $B_{b}^{3 a}=$ $(i / \sqrt{2}) \sigma_{b}^{a}$. We substitute this value in (3.8d). As a result, we have

$$
\sigma_{b}^{a}=i \sqrt{2} B_{3 b}^{a}
$$

Now, we use the relation for the Kirichenko structure tensors of six-dimensional Hermitian submanifolds of Cayley algebra [2, 4]:

$$
B_{\gamma}^{\alpha \beta}=\frac{1}{\sqrt{2}} \varepsilon^{\alpha \beta \mu} D_{\mu \gamma}, \quad B_{\alpha \beta}^{\gamma}=\frac{1}{\sqrt{2}} \varepsilon_{\alpha \beta \mu} D^{\mu \gamma},
$$

where

$$
D_{\mu \gamma}= \pm T_{\mu \gamma}^{8}+i T_{\mu \gamma}^{7}, \quad D^{\mu \gamma}=D_{\hat{\mu} \hat{\gamma}}= \pm T_{\hat{\mu} \hat{\gamma}}^{8}-i T_{\hat{\mu} \hat{\gamma}}^{7} .
$$


Here $T_{k j}^{\varphi}$ are components of the configuration tensor (in Gray's notation [9], or of the Euler curvature tensor [7]) of the Hermitian submanifold $M^{6} \subset O ; \varepsilon^{\alpha \beta \mu}=$ $\varepsilon_{123}^{\alpha \beta \mu}$ and $\varepsilon_{\alpha \beta \mu}=\varepsilon_{\alpha \beta \mu}^{123}$ are components of the third-order Kronecher tensor [14]. From (3.8a), we obtain

$$
B_{c}^{a b}=0 \Longleftrightarrow \frac{1}{\sqrt{2}} \varepsilon^{a b \gamma} D_{\gamma c}=0 \Longleftrightarrow \frac{1}{\sqrt{2}} \varepsilon^{a b 3} D_{3 c}=0 \Longleftrightarrow D_{3 c}=0
$$

The same reasoning can be applied in reference to the condition

$$
B_{3}^{a b}=0
$$

to obtain

$$
B_{3}^{a b}=0 \Longleftrightarrow \frac{1}{\sqrt{2}} \varepsilon^{a b y} D_{\gamma 3}=0 \Longleftrightarrow \frac{1}{\sqrt{2}} \varepsilon^{a b 3} D_{33}=0 \Longleftrightarrow D_{33}=0
$$

So, $D_{3 c}=D_{33}=0$, that is,

$$
D_{3 \alpha}=0
$$

From (3.8e), we get

$$
\sigma_{3}^{b}=\sigma_{3 \hat{b}}=-i B_{3}^{3 b}=-i \frac{1}{\sqrt{2}} \varepsilon^{3 b y} D_{\gamma 3}=0
$$

We have $\sigma_{a b}=\sigma_{\hat{a} \hat{b}}=\sigma_{3 b}=\sigma_{3 \hat{b}}=0$. Using (3.8b), we will compute the rest of the components of the second fundamental form

$$
\sigma_{\hat{a} b}=\sigma_{b}^{a}=i \sqrt{2} B_{b}^{a 3}=i \sqrt{2} \frac{1}{\sqrt{2}} \varepsilon^{a 3 \gamma} D_{\gamma b}=i \varepsilon^{a 3 c} D_{c b} .
$$

Then

$$
\begin{aligned}
& \sigma_{\hat{1} 1}=i \varepsilon^{13 c} D_{c 1}=i \varepsilon^{132} D_{21}=-i D_{21}, \\
& \sigma_{\hat{1} 2}=i \varepsilon^{13 c} D_{c 2}=i \varepsilon^{132} D_{22}=-i D_{22}, \\
& \sigma_{\hat{2} 1}=i \varepsilon^{23 c} D_{c 1}=i \varepsilon^{231} D_{11}=+i D_{11}, \\
& \sigma_{\hat{2} 2}=i \varepsilon^{23 c} D_{c 2}=i \varepsilon^{231} D_{12}=+i D_{12}, \\
& \sigma_{1 \hat{1}}=\overline{\sigma_{\hat{1} 1}}=i D^{12}, \\
& \sigma_{1 \hat{2}}=\overline{\sigma_{\hat{1} 2}}=i D^{22}, \\
& \sigma_{2 \hat{1}}=\overline{\sigma_{\hat{2} 1}}=-i D^{11}, \\
& \sigma_{2 \hat{2}}=\overline{\sigma_{\hat{2} 2}}=-i D^{12} .
\end{aligned}
$$


We obtain that the matrix of the second fundamental form of the immersion of the cosymplectic hyperspace $N$ into $M^{6} \subset O$ looks as follows:

$$
\sigma=\left(\begin{array}{ccccc}
0 & 0 & 0 & i D^{12} & -i D^{11} \\
0 & 0 & 0 & i D^{22} & -i D^{12} \\
0 & 0 & \sigma_{33} & 0 & 0 \\
-i D_{12} & -i D_{22} & 0 & 0 & 0 \\
i D_{11} & i D_{22} & 0 & 0 & 0
\end{array}\right) .
$$

Using, from [2], the identities

$$
D^{11} D^{22}=\left(D^{12}\right)^{2}, \quad D_{11} D_{22}=\left(D_{12}\right)^{2},
$$

we obtain that the matrices

$$
\left(\begin{array}{cc}
-i D_{12} & -i D_{22} \\
i D_{11} & i D_{12}
\end{array}\right), \quad\left(\begin{array}{cc}
i D^{12} & -i D^{11} \\
i D^{22} & -i D^{12}
\end{array}\right)
$$

are degenerate. Therefore the matrix $\sigma$ is also degenerate (rank $\sigma \leq 3$ ).

Hence, we have obtained the following result.

THEOREM 3.1. Every cosymplectic hypersurface of a six-dimensional Hermitian submanifold of Cayley algebra is a ruled manifold.

Studying the matrix $\sigma$, we come to another result. Indeed, the criterion of the minimality of the hypersurface is the following identity [1, 2]:

$$
g^{k j} \sigma_{k j}=0
$$

Knowing how the matrix of the metric tensor looks [15],

$$
\left(\begin{array}{lllll}
0 & 0 & 0 & 1 & 0 \\
0 & 0 & 0 & 0 & 1 \\
0 & 0 & 1 & 0 & 0 \\
1 & 0 & 0 & 0 & 0 \\
0 & 1 & 0 & 0 & 0
\end{array}\right),
$$

we have

$$
\begin{aligned}
g^{k j} \sigma_{k j} & =g^{a b} \sigma_{a b}+g^{\hat{a} \hat{b}} \sigma_{\hat{a} \hat{b}}+g^{\hat{a} b} \sigma_{\hat{a} b}+g^{a \hat{b}} \sigma_{a \hat{b}}+g^{33} \sigma_{33} \\
& =i D_{12}-i D_{12}+i D^{12}-i D^{12}+\sigma_{33}=\sigma_{33} .
\end{aligned}
$$

That is why $g^{k j} \sigma_{k j}=0 \Leftrightarrow \sigma_{33}=0$. The equality $\sigma_{33}=0$ means that $\sigma(\xi, \xi)=0$. Thus we have proved the following theorem.

THEOREM 3.2. The cosymplectic hypersurface of a six-dimensional Hermitian submanifold of Cayley algebra is minimal if and only if its second fundamental form satisfies the condition $\sigma(\xi, \xi)=0$. 
As the hypersurface $N$ is a totally geodesic submanifold of a Hermitian submanifold $M^{6} \subset O$ precisely when the matrix $\sigma$ vanishes, we can conclude that the conditions

$$
D_{11}=D_{12}=D_{22}=D^{11}=D^{12}=D^{22}=\sigma_{33}=0
$$

form a criterion for $N$ to be a totally geodesic submanifold of $M^{6}$.

We recall [5] that the almost Hermitian manifold satisfies the $g$-cosymplectic hypersurfaces axiom if through every point of this manifold passes a totally geodesic cosymplectic hypersurface. That is why for the Hermitian submanifold $M^{6} \subset O$, satisfying the $g$-cosymplectic hypersurfaces axiom, the equalities (3.27) hold at every point of $M^{6}$. As we have proved previously [2, 4], the matrix $\left(D_{k j}\right)$ of a six-dimensional Hermitian submanifold of the octave algebra looks as follows:

$$
D=\left(\begin{array}{cccccc}
D_{11} & D_{12} & D_{13} & 0 & 0 & 0 \\
D_{21} & D_{22} & D_{23} & 0 & 0 & 0 \\
D_{31} & D_{32} & D_{33} & 0 & 0 & 0 \\
0 & 0 & 0 & D^{11} & D^{12} & D^{13} \\
0 & 0 & 0 & D^{21} & D^{22} & D^{23} \\
0 & 0 & 0 & D^{31} & D^{32} & D^{33}
\end{array}\right)
$$

If $M^{6}$ satisfies the $g$-cosymplectic hypersurfaces axiom, then, taking into account (3.17) and (3.27), we get that this matrix vanishes. But the matrix $\left(D_{k j}\right)$ vanishes at every point of a six-dimensional almost Hermitian submanifold of the Cayley algebra precisely when the given submanifold is Kählerian [2, 3, 4, 12]. That is why we have the following theorem.

THEOREM 3.3. Every six-dimensional Hermitian submanifold of Cayley algebra satisfying the $g$-cosymplectic hypersurfaces axiom is a Kählerian manifold.

\section{REFERENCES}

[1] O. E. Arsen'eva and V. F. Kirichenko, Self-dual geometry of generalized Hermitian surfaces, Mat. Sb. 189 (1998), no. 1, 21-44 (Russian).

[2] M. B. Banaru, Hermitian Geometry of Six-Dimensional Submanifolds of Cayley Algebra, MSPU, Moscow, 1993.

[3] _ On Gray-Hervella classes of almost Hermitian manifolds on sixdimensional submanifolds of Cayley algebra, Sci. Works of MSPU (1994), 36-38.

[4] __ On almost Hermitian structures induced by means of 3-vector cross products on six-dimensional submanifolds of the octave algebra, Polyanalytical Functions, Smolensk State Pedagogical University (SGPU), Smolensk, 1997, pp. 113-117.

[5] _ G-cosymplectic hypersurfaces axiom and six-dimensional Hermitian submanifolds of the octave algebra, Problems of Complex Analysis 2 (2000), 36-41.

[6] _ On Kirichenko tensors, Problems of Complex Analysis 2 (2000), 42-48. 
[7] É. Cartan, Riemannian Geometry in an Orthogonal Coordinate System, Izdat. Moskov. Univ., Moscow, 1960 (Russian).

[8] S. Goldberg, Totally geodesic hypersurfaces of Kaehler manifolds, Pacific J. Math. 27 (1968), 275-281.

[9] A. Gray, Some examples of almost Hermitian manifolds, Illinois J. Math. 10 (1966), 353-366.

[10] _ Vector cross products on manifolds, Trans. Amer. Math. Soc. 141 (1969), 465-504.

[11] V. F. Kirichenko, On nearly-Kählerian structures induced by means of 3-vector cross products on six-dimensional submanifolds of Cayley algebra, Vestnik MGU 3 (1973), 70-75.

[12] _ Classification of Kähler structures induced by vector triple products on six-dimensional submanifolds of the Cayley algebra, Izv. Vyssh. Uchebn. Zaved. Mat. (1980), no. 8, 32-38 (Russian).

[13] V. F. Kirichenko and L. V. Stepanova, On the geometry of hypersurfaces of quasiKählerian manifolds, Uspekhi Mat. Nauk 50 (1995), no. 2, 213-214.

[14] A. Lichnerowicz, Théorie Globale des Connexions et des Groupes d'Holonomie, Cremonese, Rome, 1955 (French).

[15] L. V. Stepanova, Contact Geometry of Hypersurfaces of Quasi-Kählerian Manifolds, MSPU, Moscow, 1995.

[16] _ Q Quasi-Sasakian structure on hypersurfaces of Hermitian manifolds, Sci. works of MSPU (1995), 187-191.

[17] Y. Tashiro, On contact structure of hypersurfaces in complex manifolds. I, Tôhoku Math. J. (2) 15 (1963), 62-78.

Ahmad Al-Othman: Department of Mathematics, Applied Science University, Amman 11931, Jordan

M. Banaru: Department of Computer Technologies, Smolensk University of Humanities, Smolensk 214014, Russia

E-mail address: banaru@shu.ru 


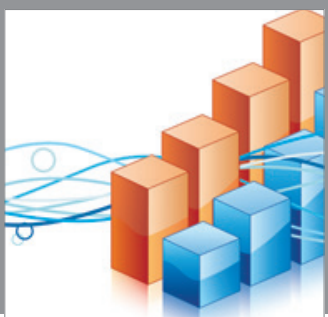

Advances in

Operations Research

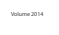

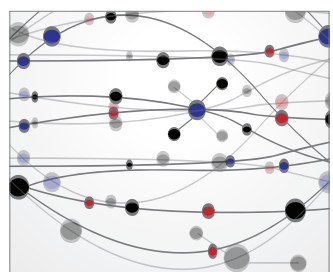

\section{The Scientific} World Journal
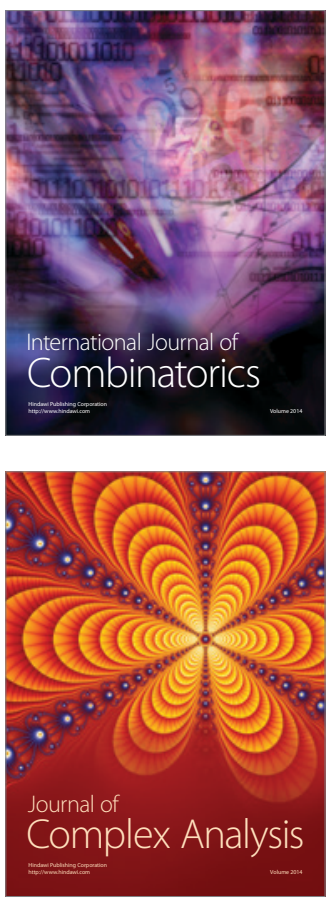

International Journal of

Mathematics and

Mathematical

Sciences
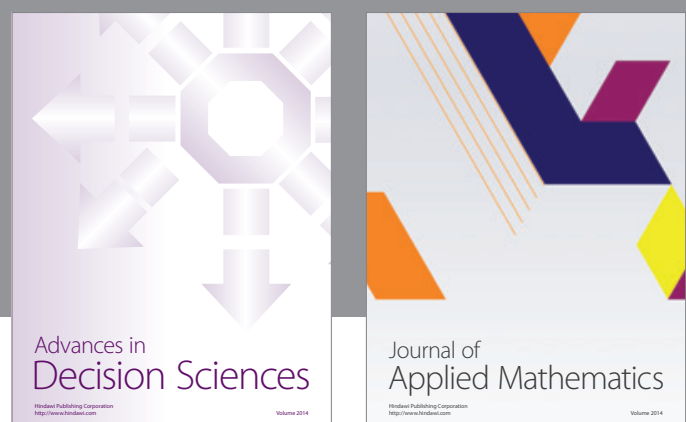

Journal of

Applied Mathematics
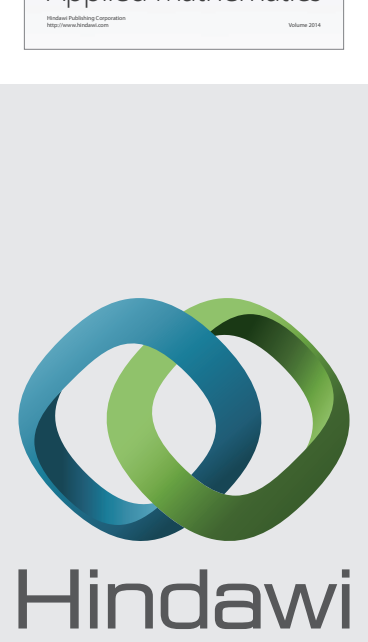

Submit your manuscripts at http://www.hindawi.com
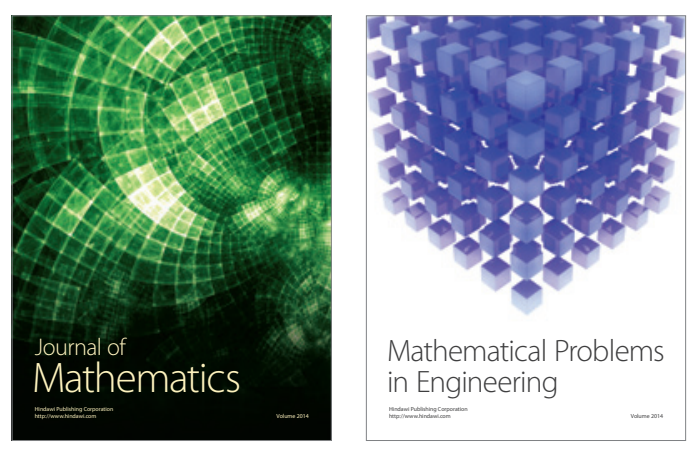

Mathematical Problems in Engineering
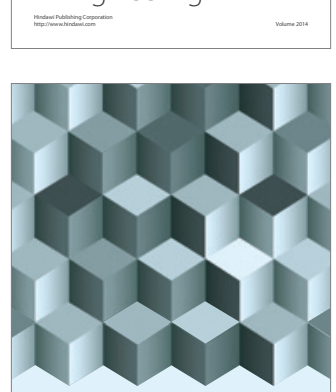

Journal of

Function Spaces
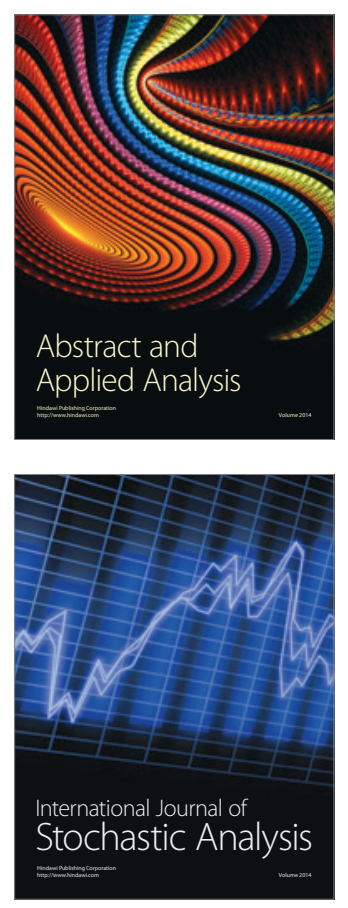

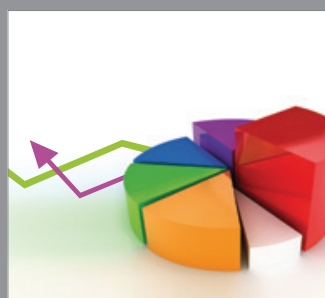

ournal of

Probability and Statistics

Promensencen
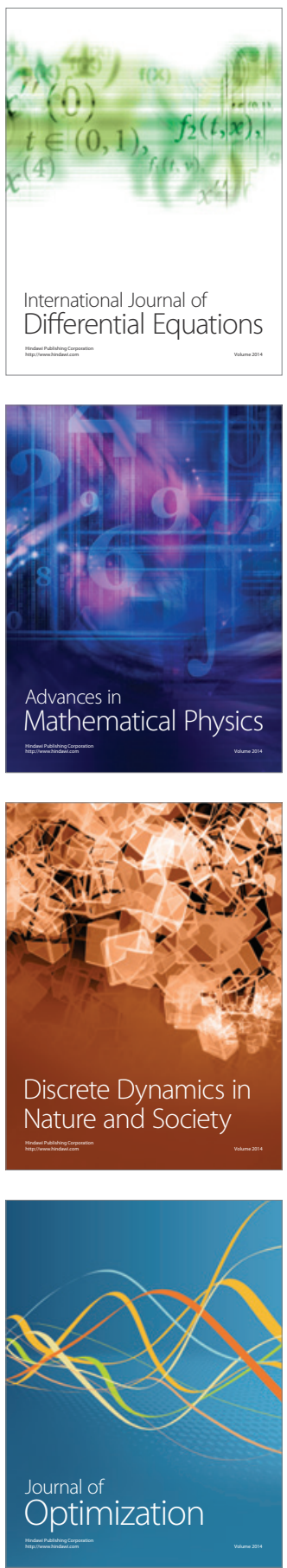